\title{
Analysis of Non-Steady Stage in SAW Gas Sensors with Semiconducting Sensor Layers
}

\author{
M. URBAŃCZYK ${ }^{a, *}$ AND T. HeJCZYK ${ }^{b}$ \\ ${ }^{a}$ Faculty of Electrical Engineering, Silesian University of Technology, B. Krzywoustego 2, 44-100 Gliwice, Poland \\ ${ }^{b}$ ENTE Sp. z o.o., Gaudiego 7, 44-100 Gliwice, Poland
}

\begin{abstract}
The profile of the gas concentration in the sensor layer can be expressed as a polynomial function involving the diffusion coefficient $\left(D_{\mathrm{K}}\right)$, semiconductor film thickness $(h)$, rate constant $(k)$, gas concentration outside the semiconductor film $\left(C_{\mathrm{S}}\right)$. Before reaching a steady state of the concentration profile, its behavior depends on a few factors as the distance from the piezoelectric surface, the rate constant, the thickness of the layer and the diffusion constant and time. We are going to simulate temporary processes in the semiconductor sensor film in the surface acoustic wave gas sensor system and to describe the influence on relative changes of the surface acoustic wave velocity. The numerical results basing on the code written in Pyton, are described and analyzed.
\end{abstract}

PACS: 07.07.Df, 43.25.Fe, 68.35.Iv, 77.65.Dq

\section{Introduction}

The generally applied gas sensors, basing on electrochemical, photoacoustic or gas chromatography, do not warrant a sufficient accuracy of measurements or require laborious operations in the laboratory. Therefore, presently investigations have been started concerning the structure of new cheap gas sensors with adequate metrological parameters. Previous to their production computer simulations are carried out in order to select the right structural and constructional parameters and conditions of operation of the sensor. Of no less importance is also the matching of a mathematical model to the achieved experimental results. These papers suggest a theoretical model as well as numerical analyses of a gas sensor of surface acoustic wave (SAW) type [1-10], in its steady state. As the phenomenon of the acoustoelectric effect depends in the set of a semiconducting layer and piezoelectric acoustic waveguide on the profile of the distribution of concentrations of the charge carriers in the sensor layer, it has been suggested to divide the sensor layer into thin sublayers, assuming a constant concentration of the carriers in these sublayers, alternating in each subsequent sublayer situated at different distances from the acoustic waveguide. Transforming the impedance of the respective sublayers to the surface of the waveguide, a substituting impedance "visible" for the acoustic surface wave was determined. Such a procedure permits to determine changes in the velocity of propagation of the surface wave due to changes in the concentration of charge carriers in the sensor layer, affected by the ambient gas. A stationary model will be supplemented by time depen-

* corresponding author; e-mail: murbanczyk@polsl.pl dences permitting investigations concerning the dynamic characteristics of SAW gas sensor.

\section{The influence of the diffusion of gas into sensor layer on the acoustoelectric effect}

The electric properties of the semiconducting sensor layer deposited on the piezoelectric waveguide depend on the ambient atmosphere, particularly on the concentration of active particles in the gas [11-15]. The diffusion of gas particles into the sensor layer causes the formation of a certain profile of the distribution of concentration of these particles deep into the layer. So far it has been assumed that the profile of the distribution of the particles and hence also the profile of the distribution of electric conductivity in the sensor layer are homogeneous over the whole cross-section of the layer. The phenomenon of the diffusion of gas into the sensor layer is of essential importance in the case of porous layer with a developed surface. Such layers are built up in expressly performed technological processes. A high porosity ensures a high sensitivity to the impact of gas. As far as porosity is concerned, there are to be distinguished: surface diffusion at a diameter of the pores below $1 \mathrm{~nm}$, Knudsen's diffusion when the diameter of the pores amounts to $1-100 \mathrm{~nm}$, and molecular diffusion in the case of pores with diameters exceeding $100 \mathrm{~nm}[8,9]$. Practically, Knudsen's diffusion is most essential in the application of sensors. Knudsen's diffusion is the diffusive transport of gas, taking place in pores which are so small that the mean free way of the diffusing molecules is restricted by their collision with the walls of the microspores and not by their collision with other molecules. In the case of Knudsen's diffusion the mean free way of the gas molecules is comparable with the dimensions of the pores. One of the chief 
domains of the occurrence of Knudsen's diffusion is the transport in microporous substances. This phenomenon cannot be described by means of formalism basing on averaged parameters with respect to time and space such as: pressure, density, velocity and temperature, complying with a certain set of partial differential equations.

\section{Processes of diffusion into the sensor layer}

In a sensor with a SAW the chemically active sensor layer is deposited on a piezoelectric waveguide (Fig. 1). In sensors which operate basing on the acoustoelectric phenomenon the electric conductivity of the sensor layer is affected by the ambient gas. The authors of [1] and [2] analyzed the influence of the diffusion of the ambient gas molecules into the sensor layer on changes of the conductance of this layer. This process becomes essential when the layer is porous. Of special importance is the case of Knudsen's diffusion. The profile of the concentration of the gas molecules in the layer conditioned by value of the ratio of the constant reaction rate $k$ to the coefficient of diffusion $D, k / D$ : when this value decreases, the distribution of the gas molecules in the sensor layer becomes more and more homogeneous. If the coefficient of the rate of reaction $k$ is very high or the value of the constant of diffusion $D$ is low, the gas penetrates merely the zone adjacent to the surface of the sensor layer. The constants $D$ and $k$ depend also on temperature.

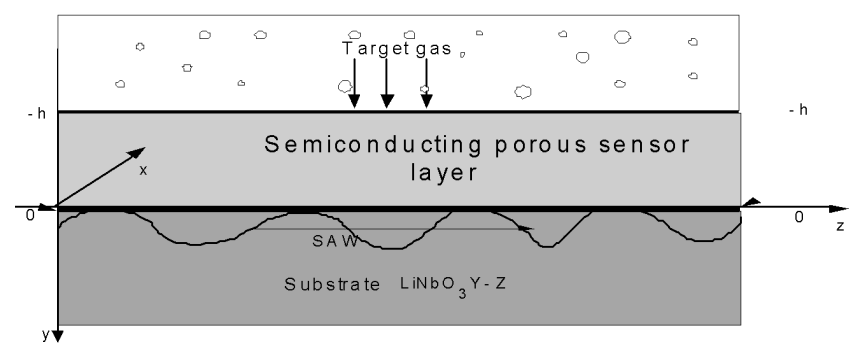

Fig. 1. Model of a gas sensor with the sensor layer on a piezoelectric substrate.

Thus, a change of temperature involves a change of the profile of the distribution of the investigated gas in the sensor layer [1]. The diffusion is also a kinetic phenomenon, depending on time. The profile of the distribution of gas molecules in the layer changes, therefore, with the lapse of time. Time analysis of this phenomenon permits to test the response and regeneration of the sensor as a function of time. The distribution of the concentration of gas molecules in the layer is the function of time $t$ and the depth $y$ in a resistance sensor, making use of Fourier's transformat, has been dealt with by Sakai and others $[1,2]$ expressing the concentration $C(y, t)$ as follows:

$$
C(y, t)=C_{\mathrm{S}}\left\{1-\frac{2}{\pi} \sum_{n=1}^{\infty} \frac{1-(-1)^{n}}{n}\left[\exp \left(-\omega_{n}^{2} t\right)\right.\right.
$$

$$
\left.\left.+\frac{k\left(1-\left(1+\omega_{n}^{2} t\right) \exp \left(-\omega_{n}^{2} t\right)\right)}{\omega_{n}^{2}+k\left(1-\exp \left(-\omega_{n}^{2} t\right)\right)}\right] \sin \frac{n \pi(h-y)}{2 h}\right\}
$$

where $\omega_{n}=\frac{n \pi \sqrt{D}}{2 h}, C_{\mathrm{S}}$ - concentration on the surface of the sensor $(y=-h), n-$ number of iterations, $h-$ thickness of the sensor layer.

The distribution of gas molecules in the layer in time and space depends on quite a lot of parameters, as reaction rate, the constant of diffusion, time, temperature and other. The accuracy of numerical calculations depends on the number of iterations $n$. As may be seen, the profile of concentration depends strictly on the parameters of diffusion. The results obtained at various constants diffusion $D$ have been compared and proved to be convergent with the results obtained by Sakai et al. [2]. The profile presented in Figs. 2-4 concerns gas in the case of a fixed number of iterations $n=10$, a fixed time $t=0.1 \mathrm{~ns}$, a constant reaction $k=10^{8} \mathrm{~s}^{-1}$ and two values of the constants of Knudsen's diffusion $D=10^{8} \mathrm{~nm}^{2} \mathrm{~s}^{-1}$ and $D=10^{12} \mathrm{~nm}^{2} \mathrm{~s}^{-1}$. The accuracy of the method of determining the profile increases with the number of iterations, and already in the case of 10 iterations the results of calculations do not differ from each other. The authors of the present paper discovered also that, similarly as quoted in [2], the analysis of non-stationary states in time is of essential importance within the range from $t=10^{-15}$ to $10^{-6} \mathrm{~s}$. It may be assumed that stationary profile of concentration in the time from $10^{-6}$ to $10^{-1} \mathrm{~s}$ exactly coincides with the analytical solution in [2].

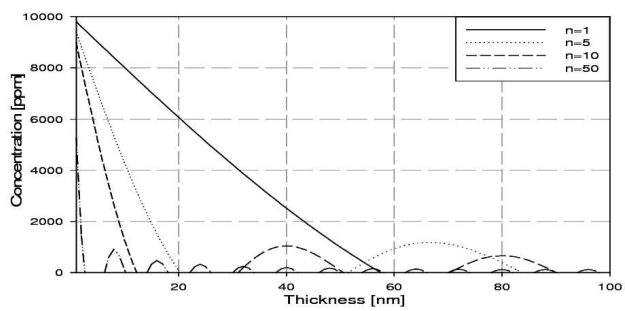

Fig. 2. Profiles of the concentration of gas depending on the number of iterations $n=1,5,10,50$, at $C_{\mathrm{S}}=$ $10000 \mathrm{ppm}, t=1 \mathrm{~ns}, k=10^{7} \mathrm{~s}^{-1}, D=10^{8} \mathrm{~nm}^{2} \mathrm{~s}^{-1}$.

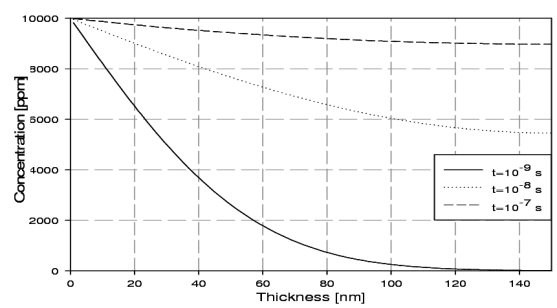

Fig. 3. Profiles of the concentration of gas depending on the thickness of the layer in the time $t=10^{-9}, 10^{-8}$, $10^{-7} \mathrm{~s}$, at $C_{\mathrm{S}}=10000 \mathrm{ppm}, n=10, k=10^{7} \mathrm{~s}^{-1}$, $D=10^{8} \mathrm{~nm}^{2} \mathrm{~s}^{-1}$. 


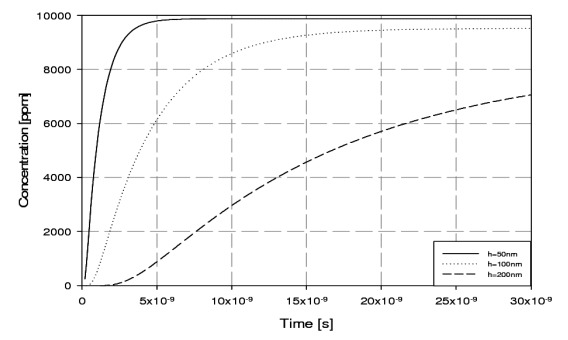

Fig. 4. Profiles of the concentration of gas in time in the case of various thicknesses of the sensor layer at $C_{\mathrm{S}}=1000 \mathrm{ppm}, n=10, k=10^{7} \mathrm{~s}^{-1}, D=$ $10^{12} \mathrm{~nm}^{2} \mathrm{~s}^{-1}$.

Calculations of the profile according to (1) were carried out in the software Pyton. The graphic interface of the user was designed permitting to change all the parameters of Eq. (1). The influence of the following parameters on the profile of the concentration of gas molecules in the layer was simulated: the reaction constant, the coefficient of diffusion, time and number of iterations $[1,2,7]$. Exemplary results, illustrating the shape of the profile of concentration in the sensor layer have been gathered in Figs. 2-4.

The chief aim of simulation was to comprehend the essence of the dynamic phenomena occurring in the sensor layer of the gauge SAW in the presence of gas. It has become possible to check the effect of the rate of the reaction taking place between the gas molecules and the semiconductor layer and to find out how long the transient processes do last until the profile of concentration has reached its stationary state.

An analysis of the results quoted above indicates that the profile of the concentration of gas diffusing into the sensor layer changes monotonically in time. At the fixed depth $y$ the concentration of the gas molecules increases monotonically, reaching in the course of several microseconds its steady state. After a somewhat longer time, e.g. $t=0.1$ to $100 \mathrm{~s}$, the steady profiles fully agree with profile determined basing on stationary Eq. (2) [1, 2]:

$$
C_{\mathrm{A}}=C_{\mathrm{A}, \mathrm{S}} \frac{\cosh \left(y \sqrt{k / D_{k}}\right)}{\cosh \left(h \sqrt{k / D_{k}}\right)} .
$$

This agreement proves true in spite of considerable differences in the notation of both equations. The considerations quoted above lead also to the conclusion that of practical importance will be the steady profile of concentration which has been arisen after a few microseconds after the layer had been exposed to action of gas.

The presented method of analyzing the profile of the gas molecules concentration in sensor layer may be applied in investigations of transient phenomena occurring in the SAW gas sensor. In this case the final effects are changes of the velocity of propagation of the surface wave due to the acoustoelectric effect of the electric field caused by the elastic wave in the piezoelectric material with charge carriers distributed in the sensor layer.

\section{Numerical analysis of the acoustoelectric effect in a sensor layer with the concentration profile of the gas molecules}

The acoustoelectric interaction of an acoustic wave in the piezoelectric with charge carriers in the sensor layer depends on the profile of the distribution of the carriers (gas molecules) in this layer. The distance of the charge carriers from the piezoelectric waveguide deserves special attention. This stationary phenomenon of interaction has been dealt with by Hejczyk et al. [7-10]. At present the procedure described above is applied in the analysis of the phenomenon of the non-stationary acoustoelectric effect in the SAW gas sensors. The output quantity is the relative change of the propagation velocity of the surface waves as the function of changes in the concentration of the gas molecules in sensor layer. The graphic diagram of the algorithm realizing the time analysis of the acoustoelectric effect has been presented in Fig. 5 .

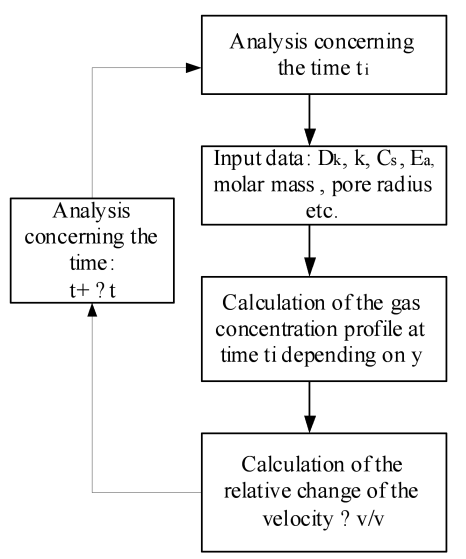

Fig. 5. Block diagram of the algorithm of the time analysis of the acoustoelectric effect in a SAW gas sensor.

The acoustoelectric interaction of the SAW with charge carriers distributed in the sensor layer was calculated in compliance with the profile resulting from diffusion of the gas molecules in the ambient atmosphere. This problem was analyzed numerically, assuming a constant concentration of the gas molecules at the surface of the sensor layer and in its surroundings. Changes of the relative velocity of the wave were determining numerically, viz. the concentration of the gas molecules on the surface $C_{\mathrm{S}}$, the values of the mean radius of the pores $r$ and the thickness of the layer $h$. The analysis was performed in the unsteady state, changing the time within the range from $t=10^{-12} \mathrm{~s}$ to $t=10^{-1} \mathrm{~s}$, basing on the assumption that after the time $t=10^{-1} \mathrm{~s}$ the profile of the concentration of the gas molecules in the sensor layer attains its steady state. The results of the analysis indicate in which way the dynamic parameters of the SAW sensor change in the presence of the given gas. The numerical analysis was carried out basing on Eqs. (1) and (3) [8, 10]: 


$$
\frac{\Delta v}{v_{0}}=-\operatorname{Re}\left(\frac{\Delta k}{k_{0}}\right)=-\frac{K^{2}}{2} \frac{\sigma_{0}^{2}\left(1+a C_{\mathrm{A}}\right)^{2}\left[1+\sum_{i=1}^{N-1} f\left(y_{i}, \sigma\left(y_{i}\right)\right)\right]^{2}}{\sigma_{0}^{2}\left(1+a C_{\mathrm{A}}\right)^{2}\left[1+\sum_{i=1}^{N-1} f\left(y_{i}, \sigma\left(y_{i}\right)\right)\right]^{2}+\left[1+\sum_{i=1}^{N-1} g\left(y_{i}, \sigma\left(y_{i}\right)\right)\right]^{2}\left(v_{0} C_{\mathrm{S}}\right)^{2}},
$$

where $C_{\mathrm{S}}=\varepsilon_{0}+\varepsilon_{p}^{\mathrm{T}}, \varepsilon_{0}$ - electrical permittivity of the vacuum (the medium above the sensor), $\varepsilon_{p}^{\mathrm{T}}$ - electrical permittivity of the piezoelectric waveguide, $N$ - number of the separation of sensor layer into sublayers, $\sigma_{0}$ surface conductivity of the sublayer.

\section{Results of the numerical analysis}

The results of the numerical analysis may serve as the basis for the optimization of the parameters of the sensor layer with respect to the maximum dynamics and sensitivity of the sensor. Figures 6-9 present exemplary results of the analysis in unsteady state concerning the sensor layer of $\mathrm{WO}_{3}$. The chosen representative gas was hydrogen, the properties of which have been investigated comprehensively in [3-7].

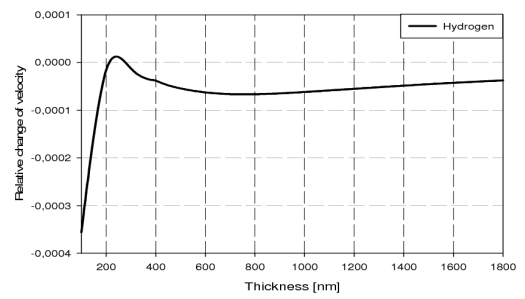

Fig. 6. Relative change of the SAW velocity. Sensor layer $\mathrm{WO}_{3}$, concentration $\mathrm{H}_{2} C_{\mathrm{S}}=300 \mathrm{ppm}, n=10$, $D=10^{12} \mathrm{~nm}^{2} \mathrm{~s}^{-1}, k=10^{8} \mathrm{~s}^{-1}, t=0.1 \mathrm{~ns}$ depending on thickness of the layer, result of the excess of the characteristics of the concentration profile.

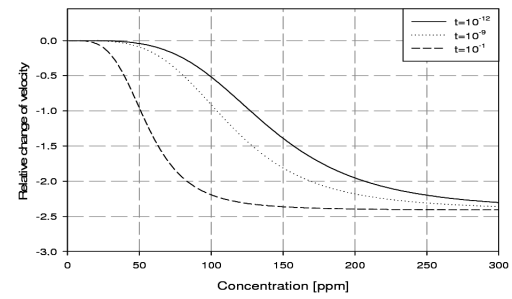

Fig. 7. The effect of the concentration of $\mathrm{H}_{2}$ molecules on the change of the SAW velocity. The calculations concern: $\sigma_{\mathrm{s}}=v_{0} C_{S}=4.7 \times 10^{-8} \Omega^{-1}, a=0.5 \mathrm{ppm}^{-1}$, thickness of the $\mathrm{WO}_{3}$ sensor layer $100 \mathrm{~nm}$, temperature $300 \mathrm{~K}$, radius of the pores $5 \mathrm{~nm}$, reaction rate $k=$ $10^{8} \mathrm{~s}^{-1}$, variable time from $t=10^{-12} \mathrm{~s}$ to $t=10^{-1} \mathrm{~s}$.

As can be seen in Fig. 9, the optimal thickness of the sensor layer is that in which the acoustoelectric effect (change of the SAW velocity) attains its maximum in steady state [7] and in the unsteady state.

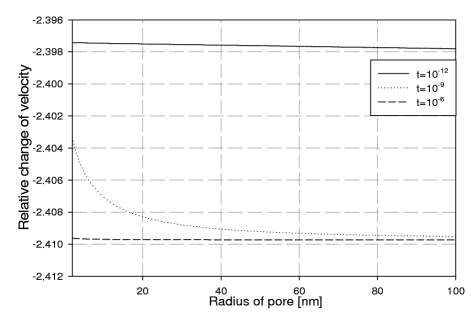

Fig. 8. The effect of the radius of the pores in the sensor layer on changes in the SAW velocity concerning $\mathrm{H}_{2}$ molecules with a concentration of $300 \mathrm{ppm}$. The calculations concern: $\sigma_{\mathrm{s}}=v_{0} C_{S}=4.7 \times 10^{-8} \Omega^{-1}$, $a=0.5 \mathrm{ppm}^{-1}$, thickness of the $\mathrm{WO}_{3}$ layer $h=100 \mathrm{~nm}$, temperature $300 \mathrm{~K}$, time from $t=10^{-12} \mathrm{~s}$ to $t=10^{-6} \mathrm{~s}$, $k=10^{8} \mathrm{~s}^{-1}$.

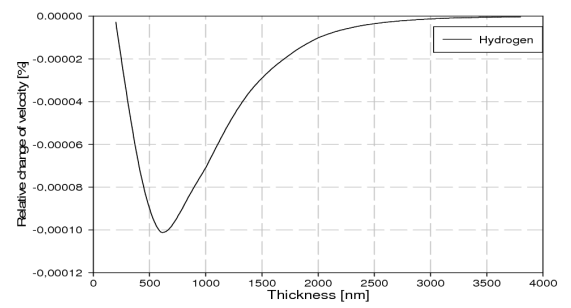

Fig. 9. The effect of the thickness of the $\mathrm{WO}_{3}$ sensor layer on changes of the SAW velocity concerning $\mathrm{H}_{2}$ molecules with concentration of $300 \mathrm{ppm}$. The calculations concern: $\sigma_{\mathrm{s}}=v_{0} C_{S}=4.7 \times 10^{-8} \Omega^{-1}, a=$ $0.5 \mathrm{ppm}^{-1}$, temperature $T=300 \mathrm{~K}$, sensor layer thickness $h=100 \mathrm{~nm}$, radius of the pores $r=2 \mathrm{~nm}$, time $t=10^{-9} \mathrm{~s}, n=100, k=10^{12} \mathrm{~s}^{-1}, D=10^{12} \mathrm{~nm}^{2} \mathrm{~s}^{-1}$.

\section{Conclusions}

The boundary conditions are disturbed when in the sensor layers are applied which conduct electrical currents [7]. Such an effect is due to the acoustoelectric effect of interaction of SAW with charge carriers in the sensor layer. The presented numerical model illustrates the acoustoelectric effect in such a layer, taking into account the profile of concentration changing in time.

Assuming the mechanism of diffusion of the Knudsen type concerning gas molecules in porous sensor layer, the profiles of the concentration of gas molecules in the layer changing in time were determined. The profiles in the unsteady state have been simulated basing on diffusion-reaction equations. Relative changes in the velocity of the surface wave were determined as the result of the acoustoelectric effect depending on numerous parameters of the sensor and its environment. 
Transient states are distinctly visible in the range of time from $10^{-12}$ to $10^{-6} \mathrm{~s}$. The performed analyses indicate that the steady state of responses of the sensor is achieved after only of a few milliseconds, depending on the parameters of the sensor layer. The calculations are also visibly affected by the iterative parameter $n$ conditioning the accuracy of numerical calculations. Practically, in calculations of the response of the sensor the number of iterations may be assumed to be 10 .

\section{Acknowledgments}

The work was partially financed within the grant No. $3742 / \mathrm{B} / \mathrm{T} 02 / 2009 / 37$.

\section{References}

[1] T. Pisarkiewicz, Mikrosensory gazów, AGH, Kraków 2007 (in Polish).

[2] G. Sakai, N. Matsunaga, E. Shimanoe, N. Yamazoe, Sensors Actuators B 80, 125 (2001).

[3] W. Jakubik, M. Urbańczyk, E. Maciak, T. Pustelny, Acta Phys. Pol. A 116, 315 (2009).
[4] W. Jakubik, Sensors Actuators B 96, 321 (2003).

[5] W. Jakubik, Thin Solid Films 515, 8345 (2007).

[6] M. Urbańczyk, SAW Gas Sensors, SUT, Gliwice 2011 (in Polish).

[7] T. Hejczyk, M. Urbanczyk, W. Jakubik, Acta Phys. Pol. A 118, 1153 (2010).

[8] T. Hejczyk, M. Urbanczyk, W. Jakubik, Acta Phys. Pol. A 118, 1148 (2010).

[9] T. Hejczyk, M. Urbanczyk, W. Jakubik, Acta Phys. Pol. A 118, 1158 (2010).

[10] M. Urbańczyk, Proc. CMEM11 48, 229 (2011).

[11] T. Pustelny, I. Zielonka, C. Tyszkiewicz, P. Karasiński, B. Pustelny, Opto-electron. Rev. 14, 161 (2006).

[12] B. Pustelny, T. Pustelny, Acta Phys. Pol. A 116, 383 (2009).

[13] T. Pustelny, A. Opilski, B. Pustelny, Acta Phys. Pol. A 114, A183 (2008).

[14] W. Jakubik, M. Urbańczyk, E. Maciak, T. Pustelny, Bull. Pol. Acad. Sci. Techn. Sci. 56, 133 (2008).

[15] T. Pustelny, J. Ignac-Nowicka, Z. Opilski, Optica Applicata 34, 249 (2004). 